\title{
Effectively Acquiring and Using Feedback
}

\author{
David M. Naeger, MD, Aaron Jen, BA, Bren Ahearn, MA, Emily M. Webb, MD
}

\section{DESCRIPTION OF THE PROBLEM}

Feedback is a vital component of medical education $[1,2]$. Feedback can hone an educator's teaching skills, guide the evolution of a curriculum, and improve the experience of students $[3,4]$. However, feedback systems are easily hampered by pitfalls and often implemented without sufficient scrutiny or review [5].

When feedback is not collected properly, it can be difficult to use. For example, collecting too little feedback can provide insufficient information, and too much feedback (or feedback that has accumulated) can be overwhelming. Delays in receiving or processing feedback limit the likelihood of effecting timely change.

When feedback is of poor quality, its utility is decreased [6]. The biggest cause of poor feedback is "feedback fatigue" [7], soliciting feedback so frequently that participation drops and the results are terse and prone to response bias. Additionally, overly vague or specific feedback has been shown to be less effective [6], as has exclusively positive or exclusively negative feedback [8]. Off-the-cuff verbal feedback seems to lack critical analysis, compared with written feedback [9].

Finally, actually doing something with the feedback is required for it to be effective. Commonly, the energy, time, or motivation required to make improvements is lacking, despite the availability of sufficient feedback. Some studies have suggested that physicians, as a group, often tend to overestimate their performance [10], thereby limiting their willingness to change. In some cases, feedback must be combined with mentorship to effect meaningful change $[5,10,11]$.

Herein, we discuss a multiyear process of critically reviewing and improving our feedback system in the largest course offered by our department-the core medical student radiology elective, in which approximately 100 students enroll per year. In particular, we focus on how our feedback systems could improve the instruction provided, while promoting educators and the educational program.

\section{WHAT WAS DONE}

\section{Definition of Feedback Goals}

Our first step was to create a list of goals for our feedback system. Through a collaborative process, all members of the radiology elective's leadership determined the characteristics of an effective feedback system, which were as follows:

1. All requested feedback should be useful (ie, actionable):

- No redundant questions;

- No questions about things that cannot be changed;
- Questions specifically assessing a targeted area of concern should be removed after sufficient information has been acquired.

2. Feedback should be required of all students, to ensure a representative set of comments and codify the expectation that everyone is responsible for helping improve the educational experience.

3. Feedback should be acquired in a timely manner, ideally the same day as the activity.

4. Feedback should be reviewed in a timely manner, ideally within a few days.

5. Feedback should result in changes (except when the feedback indicates the current state is as good as can reasonably be achieved).

6. Positive feedback should be used to support and promote educators and the educational program.

To achieve the preceding goals, the leadership decided that both daily feedback and "end-of-course" feedback were needed.

\section{Acquiring Daily Feedback}

Students were asked to provide constructive daily feedback on all activities, via the course's online learning portal. To minimize "feedback fatigue," the daily online form was parsimonious, including a required attendance verification question and only 2 
questions about the lecture itself: a 1-10 rating and a free-form comment box. A representative form is provided in Figure 1 (available online).

Completing the entire form was compulsory. Students were asked to complete the form within 24 hours. At the 24-hour mark, incomplete evaluations prompted a reminder e-mail to be sent by the course coordinator.

\section{Using Daily Feedback}

The mean score for the session, and all deidentified comments, were e-mailed to the session instructor and the course director. For most sessions, the lecturer reviewed the comments and independently considered ways to improve. In cases in which an intervention was appropriate, particularly for more-junior educators, the course director contacted the lecturer directly to discuss the issues.

\section{Acquiring End-of-Course Feedback}

Students were asked to provide general feedback on the last day of the course. The core questions that were asked, and the general structure of the survey, are presented in Figure 2 (available online). The endof-course feedback form often included questions directed at new or experimental components of the elective, such as a new radiation safety curriculum that was added. These questions were removed as soon as feedback was sufficient to guide the course director. The students were encouraged to complete the form on the last day of the course, although they were given until the subsequent business day to do so before a reminder e-mail was sent.

\section{Using End-of-Course Feedback}

Once all feedback was received, the course director and coordinator reviewed the feedback. Potential changes were discussed during the following week among the course leadership. Occasionally, changes required weeks to months to implement. Examples of changes made include removing a component of the curriculum, and increasing or decreasing the time spent on an activity.

\section{Circling Back}

A critical, but often overlooked, step in the feedback system is "circling back" to those who provided the feedback, to show them that the feedback was helpful. This circling back can be direct, ie, showing the change to those who provided the feedback, or indirect, ie, highlighting changes made in response to feedback from previous learners. Although this task is one of "public relations," this critical step helps spur current learners to provide meaningful feedback. A well-documented finding is that students often feel their feedback is not taken seriously [12]; in our experience, nothing harms feedback quality more than the sense that the feedback is not used or valued.

Throughout the course, the course director and coordinator emphasized changes made with the help of previous feedback, most notably during the course orientation. Additionally, the course director frequently referenced recent comments (anonymously) to show that the feedback is reviewed. We cannot emphasize enough the importance of circling back.

\section{Using Feedback to Promote Educators}

Our feedback is primarily obtained to improve educational activities. Indirectly, feedback can help educators in their quest to become better teachers and their efforts to be promoted.

For resident educators (who teach in our program with faculty supervision and mentorship), positive evaluations are mentioned in the biannual resident performance evaluation meetings. Per ACGME program requirements, senior residents are expected to "... supervise or act as consultants to and teach medical students and residents" (IV.A.5.d)(7) [13]. For faculty, positive feedback is included in educator portfolios and promotion packets. At our institution, teaching evaluations are a required component of every promotion packet.

\section{Using Feedback to Promote the Educational Program}

Educational programs need educators. Educators in academic departments, however, are notoriously overcommitted, often owing to a large burden of clinical, administrative, and research responsibilities [14]. Given that teaching is evaluated as part of the promotion process, we have found that highlighting this benefit is useful when recruiting educators. In addition, we provide annual letters to faculty members, delineating their teaching contributions and a summary of student comments. This information is formatted for easy importing into faculty CVs. 


\section{OUTCOMES}

\section{Daily Feedback}

Between September 2013 and April 2015 , approximately 6,700 individual comments were obtained. Eighty-four percent of the comments were collected the same day as the session, thereby meeting the goal of within $<24$ hours. Eighty-six percent of the comments were provided to the educators within five working days.

Daily feedback was estimated to lead to substantial changes in approximately $1-2$ lectures per month. Occasionally, a session was removed entirely. Approximately 5-10 sessions were altered in minor ways, based on the feedback provided (eg, changes in presentation technique, etc.). All of the major, and half of the minor, changes were prompted by, guided by, or achieved with the help of the course director. Changes were more common in new lectures and those given by more-junior lecturers.

\section{End-of-Course Feedback}

From September 2013 to April 2015, a total of 143 end-of-course feedback forms were acquired during the course of 10 sessions. Approximately $85 \%$ of the students completed the form the same day the course ended. Comments were reviewed by the course director and the course coordinator within 1 day for $100 \%$ of the sessions.

Approximately $80 \%$ of the feedback episodes led to minor changes to the course before the start of the next session. Major changes occurred after $20 \%$ of the feedback episodes, although the decision to make a major change was usually the result of repeated similar comments over multiple sessions.

Changes resulting from end-ofcourse feedback include:

- Better sequencing of lectures based on topic difficulty;

- Shortening of an assignment regarding reading-room observations;

- Shortening of questionnaires that students used to review selflearning modules (fittingly, feedback helped improve our feedback process!);

- Revamping of our radiation safety curriculum;

- Better distribution of lectures throughout the course, to avoid unusually long days; and

- Limiting the number of individual announcements made to students (these are now more aggregated).

\section{Using Feedback to Promote Educators}

Feedback from teaching has been used in three iterations of diagnostic radiology residency Clinical Competency Committee meetings; the information is considered helpful and has been used to provide a more complete picture of some residents' scholarly activity. Our database has been used to create two rounds of annual teaching letters for faculty members, with 42 and 56 letters generated in each round, respectively. Faculty members have used the letters to complete their online $\mathrm{CVs}$ and have been appreciative. The teaching accomplishments of a number of our faculty members have helped in their promotions.

\section{Using Feedback to Promote the Educational Program}

The annual feedback letters, signed by the educational leaders and the department chair, have established teaching as a valued activity. We continue to enjoy strong support from our faculty members for our teaching mission.

\section{CONCLUSIONS}

We have developed a feedback system for our core radiology course that meets our outlined goals:

- A near- $100 \%$ completion rate, achieved via the use of IT and by linking feedback to the recording of course attendance;

- All feedback is reviewed in a systematic and timely manner;

- Changes based on feedback are implemented in a timely manner;

- We "circle back" to emphasize the value of feedback;

- Feedback is made readily available to faculty members and the department, and feedback is used to promote educators and the educational program.

We believe similar feedback systems can be applied to other institutions. Although our system requires a small effort to set up and upkeep, the added value is tremendous.

\section{REFERENCES}

1. Ende J. Feedback in clinical medical education. JAMA 1983;250:777-81.

2. Jones R, Higgs R, De Angelis C, Prideaux D. Changing face of medical curricula. Lancet 2001;357:699-703.

3. Marsh HW, Roche L. The use of students' evaluations and an individually structured intervention to enhance university teaching effectiveness. Am Educ Res J 1993;30: 217-51.

4. Alemoni LM. The usefulness of student evaluations in improving college teaching. Instr Sci 1978;7:95-105.

5. Richardson JTE. Instruments for obtaining student feedback: a review of the literature. Assess Eval High Educ 2005:37-41.

6. Hewson MG, Little ML. Giving feedback in medical education: verification of recommended techniques. J Gen Intern Med 1998;13:111-6.

7. Fysh T. Feedback fatigue. Clin Teach 2011;8:288. 
8. Bing-You RG, Trowbridge RL. Why medical educators may be failing at feedback. JAMA 2009;302:1330-1.

9. Colletti LM. Difficulty with negative feedback: face-to-face evaluation of junior medical student clinical performance results in grade inflation. J Surg Res 2000;90: $82-7$.

10. Davis DA, Mazmanian PE, Fordis M, Van Harrison R, Thorpe KE, Perrier L. Accuracy of physician self-assessment compared with observed measures of competence: a systematic review. JAMA 2006;296: 1094-102.

11. Kember D, Leung DYP, Kwan KP. Does the use of student feedback questionnaires improve the overall quality of teaching? Assess Eval High Educ 2002;27:411-25.

12. Spencer KJ, Schmelkin LP. Student perspectives on teaching and its evaluation. Assess Eval High Educ 2002;27:397-409.

13. ACGME: Diagnostic radiology program requirements. Available at: www.acgme. org/acgmeweb. Accessed May 1, 2015.
14. Straus CM, Webb EM, Kondo KL, et al. Medical student radiology education: summary and recommendations from a national survey of medical school and radiology department leadership. J Am Coll Radiol 2014;11:606-10.

\section{ADDITIONAL RESOURCES}

Additional resources can be found online at: http://dx.doi.org/10.1016/ j.jacr.2015.05.011

David M. Naeger, MD, Aaron Jen, BA, Bren Ahearn, MA, and Emily M. Webb, MD, are from the University of California, San Francisco, San Francisco, California.

The authors have no conflicts of interest related to the material discussed in this article.

David M. Naeger, MD: Department of Radiology and Biomedical Imaging, University of California, San Francisco, 505 Parnassus Ave, M-391, Box 0628, San Francisco, CA 94143-0628; e-mail: david.naeger@ucsf.edu. 